\title{
Visual Basic 2005
}

Recipes

A Problem-Solution Approach

Todd Herman, Allen Jones,

Matthew MacDonald, and

Rakesh Rajan 


\section{Visual Basic 2005 Recipes: A Problem-Solution Approach}

\section{Copyright $\odot 2007$ by Todd Herman, Allen Jones, Matthew MacDonald, Rakesh Rajan}

All rights reserved. No part of this work may be reproduced or transmitted in any form or by any means, electronic or mechanical, including photocopying, recording, or by any information storage or retrieval system, without the prior written permission of the copyright owner and the publisher.

ISBN-13 (pbk): 978-1-59059-852-8

ISBN-10 (pbk): 1-59059-852-0

Printed and bound in the United States of America 987654321

Trademarked names may appear in this book. Rather than use a trademark symbol with every occurrence of a trademarked name, we use the names only in an editorial fashion and to the benefit of the trademark owner, with no intention of infringement of the trademark.

Lead Editor: Ewan Buckingham

Technical Reviewer: Damien Foggon

Editorial Board: Steve Anglin, Ewan Buckingham, Gary Cornell, Jonathan Gennick, Jason Gilmore, Jonathan Hassell, Chris Mills, Matthew Moodie, Jeffrey Pepper, Ben Renow-Clarke, Dominic Shakeshaft, Matt Wade, Tom Welsh

Project Manager: Beth Christmas

Copy Edit Manager: Nicole Flores

Copy Editor: Marilyn Smith

Assistant Production Director: Kari Brooks-Copony

Production Editor: Ellie Fountain

Compositor: Susan Glinert

Proofreader: Liz Welch

Indexer: John Collin

Artist: Kinetic Publishing Services, LLC

Cover Designer: Kurt Krames

Manufacturing Director: Tom Debolski

Distributed to the book trade worldwide by Springer-Verlag New York, Inc., 233 Spring Street, 6th Floor, New York, NY 10013. Phone 1-800-SPRINGER, fax 201-348-4505, e-mail orders-ny@springer-sbm.com, or visit http://www. springeronline.com.

For information on translations, please contact Apress directly at 2855 Telegraph Avenue, Suite 600, Berkeley, CA 94705. Phone 510-549-5930, fax 510-549-5939, e-mail info@apress.com, or visit http://

WWw. apress.com.

The information in this book is distributed on an "as is" basis, without warranty. Although every precaution has been taken in the preparation of this work, neither the author(s) nor Apress shall have any liability to any person or entity with respect to any loss or damage caused or alleged to be caused directly or indirectly by the information contained in this work.

The source code for this book is available to readers at http://www. apress. com in the Source Code/Download section. 
This book is for my incredible wife and best friend Amy, as well as my son Aidan and daughter Alaina. Without them I wouldn't be the man I am today and this book may not have been possible.

- Todd Herman 


\section{Contents at a Glance}

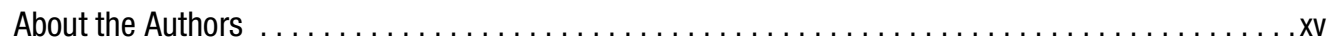

About the Technical Reviewer $\ldots \ldots \ldots \ldots \ldots \ldots \ldots \ldots \ldots \ldots \ldots \ldots \ldots \ldots \ldots \ldots \ldots \ldots \ldots \ldots \ldots$

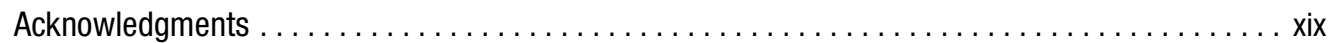

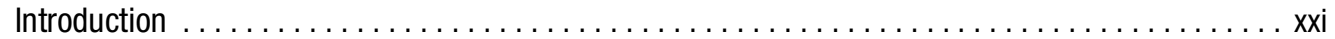

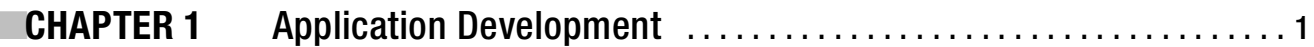

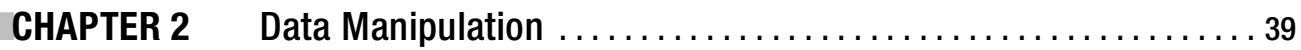

CHAPTER 3 Application Domains, Reflection, and Metadata ............. 77

CHAPTER 4 Threads, Processes, and Synchronization ............... 111

CHAPTER 5 Files, Directories, and I/0 ......................... 161

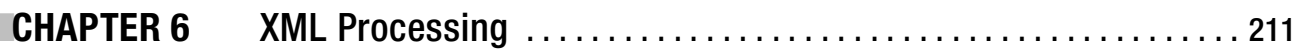

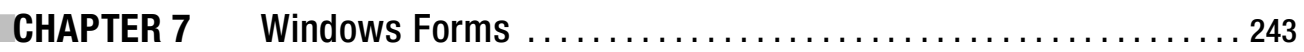

CHAPTER 8 Multimedia ..................................... 289

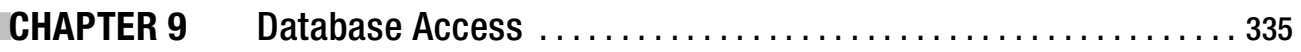

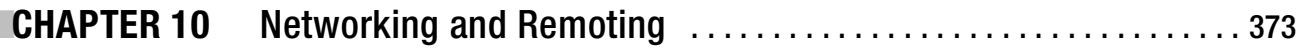

CHAPTER 11 Security and Cryptography $\ldots \ldots \ldots \ldots \ldots \ldots \ldots \ldots \ldots \ldots \ldots \ldots \ldots \ldots \ldots$

CHAPTER 12 Unmanaged Code Interoperability $\ldots \ldots \ldots \ldots \ldots \ldots \ldots \ldots . \ldots \ldots 1$

CHAPTER 13 Commonly Used Interfaces and Patterns ................ 501

CHAPTER 14 Windows Integration ............................. 545

CHAPTER 15 Language Integrated Query (LINQ) ................... 571

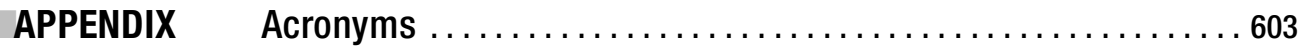

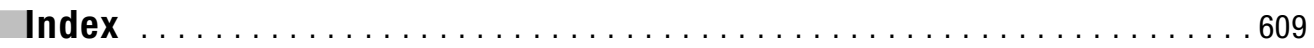




\section{Contents}

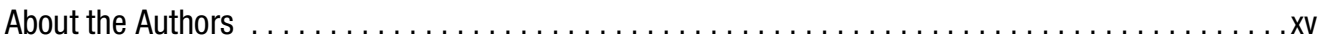

About the Technical Reviewer $\ldots \ldots \ldots \ldots \ldots \ldots \ldots \ldots \ldots \ldots \ldots \ldots \ldots \ldots \ldots \ldots \ldots \ldots \ldots \ldots \ldots \ldots$

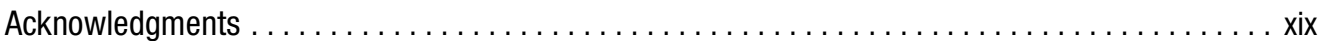

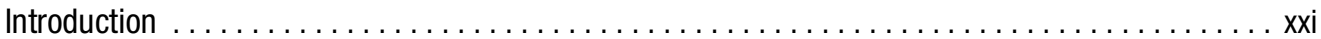

CHAPTER 1 Application Development $\ldots \ldots \ldots \ldots \ldots \ldots \ldots \ldots \ldots \ldots \ldots \ldots$

1-1. Create a Console Application from the Command Line ......... 2

1-2. Create a Windows-Based Application from the Command Line .... 4

1-3. Create and Use a Code Module from the Command Line ........ 8

1-4. Create and Use a Code Library from the Command Line ........ 10

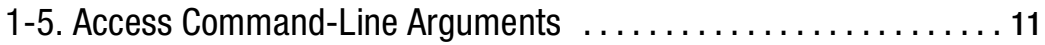

1-6. Include Code Selectively at Build Time $\ldots \ldots \ldots \ldots \ldots \ldots \ldots$

1-7. Access a Program Element That Has the Same Name

As a Keyword .................................... 17

1-8. Create and Manage Strong-Named Key Pairs . . . . . . . . . . 18

1-9. Give an Assembly a Strong Name $\ldots \ldots \ldots \ldots \ldots \ldots \ldots \ldots . \ldots . \ldots . \ldots . \ldots 19$

1-10. Verify That a Strong-Named Assembly Has Not

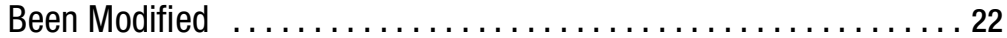

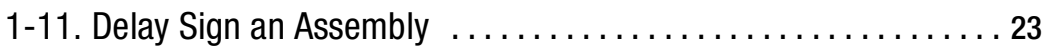

1-12. Sign an Assembly with an Authenticode Digital Signature . . . . 25

1-13. Create and Trust a Test Software Publisher Certificate ........ 29

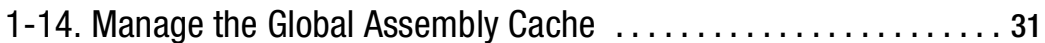

1-15. Make Your Assembly More Difficult to Decompile ........... 32

1-16. Manipulate the Appearance of the Console .............. 33

$1-17$. Embed a Resource File in an Assembly .................. 35

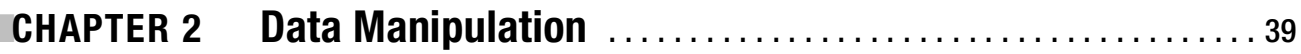

2-1. Manipulate the Contents of a String Efficiently ............. 39

2-2. Encode a String Using Alternate Character Encoding .......... 42

2-3. Convert Basic Value Types to Byte Arrays $\ldots \ldots \ldots \ldots \ldots \ldots \ldots 44$

2-4. Base64 Encode Binary Data $\ldots \ldots \ldots \ldots \ldots \ldots \ldots \ldots \ldots \ldots \ldots$

2-5. Validate Input Using Regular Expressions $\ldots \ldots \ldots \ldots \ldots \ldots \ldots$

2-6. Use Compiled Regular Expressions $\ldots \ldots \ldots \ldots \ldots \ldots \ldots \ldots \ldots$ 
2-7. Create Dates and Times from Strings $\ldots \ldots \ldots \ldots \ldots \ldots \ldots 56$

2-8. Add, Subtract, and Compare Dates and Times ............. 58

$2-9$. Sort an Array or an ArrayList .........................61

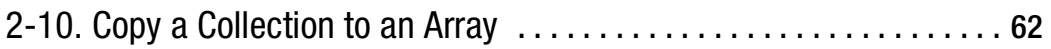

$2-11$. Use a Strongly Typed Collection $\ldots \ldots \ldots \ldots \ldots \ldots \ldots \ldots \ldots 6$

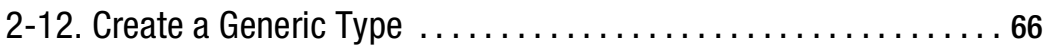

2-13. Store a Serializable 0 bject to a File $\ldots \ldots \ldots \ldots \ldots \ldots \ldots \ldots 6 . \ldots \ldots$

2-14. Read User Input from the Console $\ldots \ldots \ldots \ldots \ldots \ldots \ldots \ldots 72$

CHAPTER 3 Application Domains, Reflection, and Metadata . . . . . 77

3-1. Load an Assembly into the Current Application Domain ........ 77

3-2. Create an Application Domain ...................... 80

3-3. Execute an Assembly in a Different Application Domain ........ 82

3-4. Avoid Loading Unnecessary Assemblies into

Application Domains $\ldots \ldots \ldots \ldots \ldots \ldots \ldots \ldots \ldots \ldots \ldots \ldots$

3-5. Create a Type That Cannot Cross Application

Domain Boundaries ............................... 85

3-6. Create a Type That Can Be Passed Across Application

Domain Boundaries $\ldots \ldots \ldots \ldots \ldots \ldots \ldots \ldots \ldots \ldots \ldots \ldots$

3-7. Instantiate a Type in a Different Application Domain ......... 89

3-8. Pass Data Between Application Domains . .............. 93

3-9. Unload Assemblies and Application Domains . ............. 96

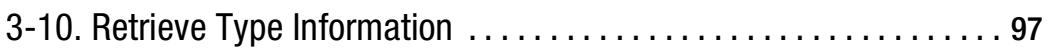

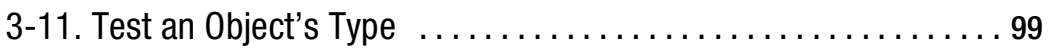

3-12. Instantiate an Object Using Reflection $\ldots \ldots \ldots \ldots \ldots \ldots \ldots 101$

3-13. Create a Custom Attribute ........................ 105

3-14. Inspect the Attributes of a Program Element

Using Reflection . . . . . . . . . . . . . . . . . . . . . . . 107

CHAPTER 4 Threads, Processes, and Synchronization $\ldots \ldots \ldots \ldots 111$

4-1. Execute a Method Using the Thread Pool $\ldots \ldots \ldots \ldots \ldots \ldots 112$

4-2. Execute a Method Asynchronously . . . . . . . . . . . . . . 115

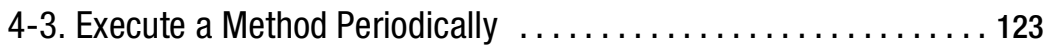

4-4. Execute a Method at a Specific Time .................. 125

4-5. Execute a Method by Signaling a WaitHandle Object ......... 127

4-6. Execute a Method Using a New Thread .................. 129

4-7. Synchronize the Execution of Multiple Threads

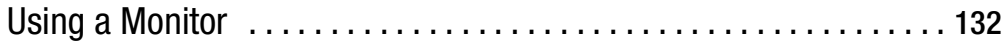


4-8. Synchronize the Execution of Multiple Threads

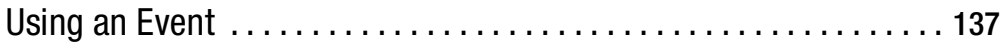

4-9. Synchronize the Execution of Multiple Threads

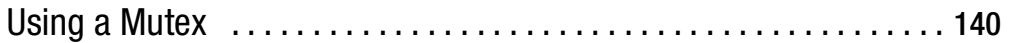

4-10. Synchronize the Execution of Multiple Threads

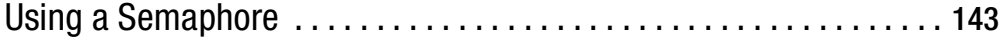

4-11. Synchronize Access to a Shared Data Value ............. 145

4-12. Know When a Thread Finishes ........................ 147

4-13. Terminate the Execution of a Thread ................... 149

4-14. Create a Thread-Safe Collection Instance ................ 151

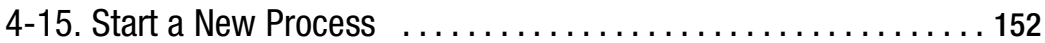

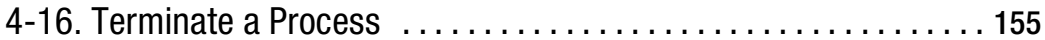

4-17. Ensure That Only One Instance of an Application Can

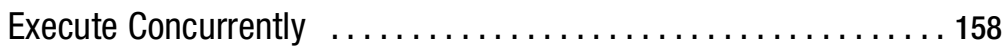

CHAPTER 5 Files, Directories, and I/0 $\ldots \ldots \ldots \ldots \ldots \ldots \ldots \ldots \ldots \ldots \ldots \ldots$

5-1. Retrieve Information About a File, Directory, or Drive $\ldots \ldots \ldots 162$

$5-2$. Set File and Directory Attributes . .................... 167

5-3. Copy, Move, or Delete a File or a Directory .............. 168

$5-4$. Calculate the Size of a Directory . . . . . . . . . . . . . . . 171

$5-5$. Retrieve Version Information for a File $\ldots \ldots \ldots \ldots \ldots \ldots \ldots \ldots \ldots$

5-6. Show a Just-in-Time Directory Tree in the TreeView Control . . . 175

$5-7$. Read and Write a Text File ........................ 177

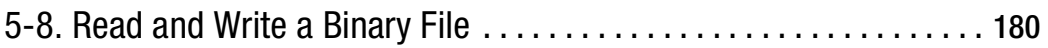

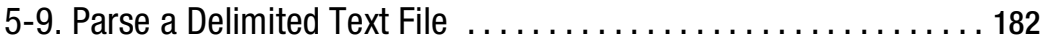

$5-10$. Read a File Asynchronously . ...................... 185

$5-11$. Find Files That Match a Wildcard Expression ............ 188

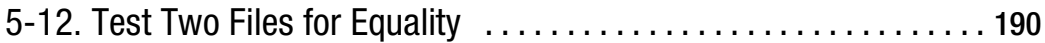

$5-13$. Manipulate Strings Representing Filenames ............. 191

$5-14$. Determine If a Path Is a Directory or a File $\ldots \ldots \ldots \ldots \ldots \ldots 193$

$5-15$. Work with Relative Paths ........................... 194

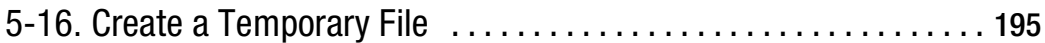

$5-17$. Get the Total Free Space on a Drive $\ldots \ldots \ldots \ldots \ldots \ldots \ldots \ldots \ldots$

$5-18$. Show the Common File Dialog Boxes $\ldots \ldots \ldots \ldots \ldots \ldots \ldots 197$

$5-19$. Use an Isolated Store ............................ 200

$5-20$. Monitor the File System for Changes . . . . . . . . . . . . 202

$5-21$. Access a COM Port ............................. 205

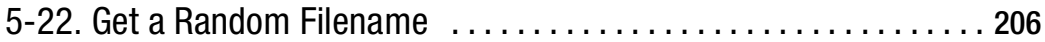

5-23. Manipulate the Access Control Lists of a File or Directory . . . . 207 


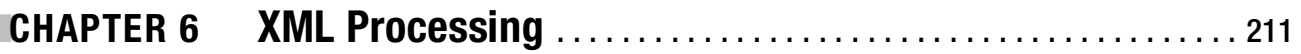

6 -1. Show the Structure of an XML Document in a TreeView ........ 211

6-2. Insert Nodes in an XML Document ................... 215

6-3. Quickly Append Nodes in an XML Document ............. 217

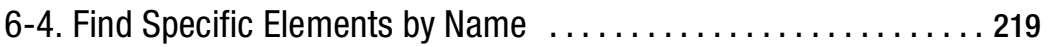

6-5. Get XML Nodes in a Specific XML Namespace ............. 221

$6-6$. Find Elements with an XPath Search $\ldots \ldots \ldots \ldots \ldots \ldots \ldots \ldots \ldots \ldots \ldots \ldots \ldots$

6-7. Read and Write XML Without Loading an Entire Document

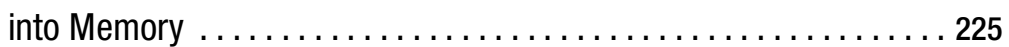

6-8. Validate an XML Document Against a Schema ............ 228

6-9. Use XML Serialization with Custom Objects $\ldots \ldots \ldots \ldots \ldots \ldots . \ldots 233$

$6-10$. Create a Schema for a .NET Class ................... 237

$6-11$. Generate a Class from a Schema .................. 237

6-12. Perform an XSL Transform ........................ 238

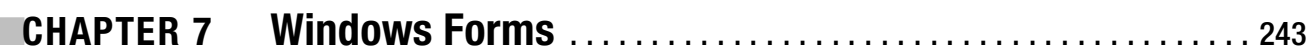

7-1. Add a Control Programmatically ................... 244

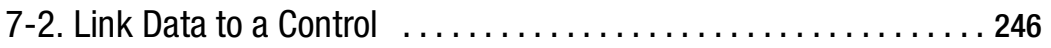

7-3. Process All the Controls on a Form $\ldots \ldots \ldots \ldots \ldots \ldots \ldots 248$

$7-4$. Track the Visible Forms in an Application ............... 249

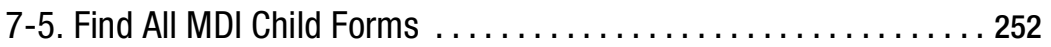

$7-6$. Save Configuration Settings for a Form ................ 255

7-7. Force a List Box to Scroll to the Most Recently Added Item . . . . 258

7-8. Restrict a Textbox to Accepting Only Specific Input .......... 259

7-9. Use an Autocomplete Combo Box .................. 262

$7-10$. Sort a List View by Any Column $\ldots \ldots \ldots \ldots \ldots \ldots \ldots \ldots \ldots . \ldots \ldots$

$7-11$. Lay Out Controls Automatically $\ldots \ldots \ldots \ldots \ldots \ldots \ldots \ldots . \ldots . \ldots . \ldots 268$

$7-12$. Use Part of a Main Menu for a Context Menu ............. 269

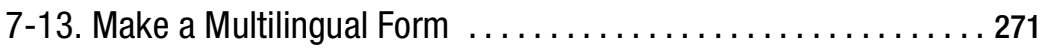

$7-14$. Create a Form That Cannot Be Moved . . . . . . . . . . . . 274

$7-15$. Make a Borderless Form Movable ................... 275

$7-16$. Create an Animated System Tray Icon ................. 277

$7-17$. Validate an Input Control ........................ 279

7-18. Use a Drag-and-Drop Operation . . . . . . . . . . . . . . 281

7-19. Use Context-Sensitive Help . . . . . . . . . . . . . . . . . . 283

7-20. Display a Web Page in a Windows-Based Application ........ 284 
$8-1$. Find All Installed Fonts $\ldots \ldots \ldots \ldots \ldots \ldots \ldots \ldots \ldots \ldots \ldots 290$

8-2. Perform Hit Testing with Shapes $\ldots \ldots \ldots \ldots \ldots \ldots \ldots \ldots \ldots 292$

8-3. Create an Irregularly Shaped Control . . . . . . . . . . . . . . 295

8-4. Create a Movable Sprite ......................... 297

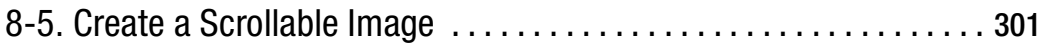

8-6. Perform a Screen Capture ........................ 303

8-7. Use Double Buffering to Increase Redraw Speed ............ 304

$8-8$. Show a Thumbnail for an Image . . . . . . . . . . . . . . . . . . . 307

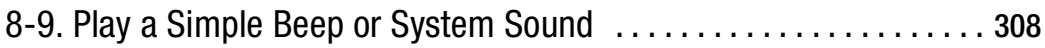

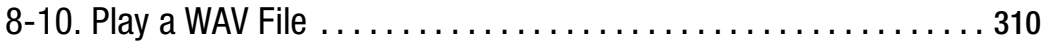

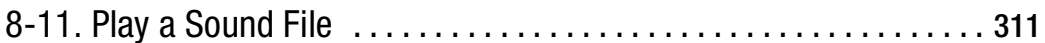

$8-12$. Show a Video with DirectShow ...................... 313

8-13. Retrieve Information About Installed Printers . ........... 316

$8-14$. Print a Simple Document ............................ 318

8-15. Print a Multipage Document ...................... 321

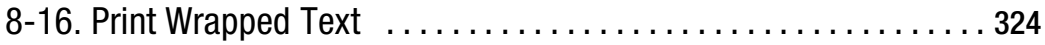

8-17. Show a Dynamic Print Preview . . . . . . . . . . . . . . . 327

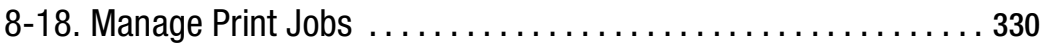

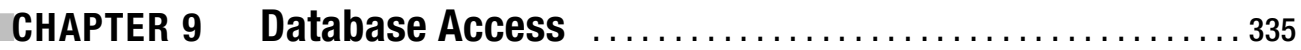

9-1. Connect to a Database .......................... 336

9-2. Use Connection Pooling .......................... 340

9-3. Create a Database Connection String Programmatically ........ 342

9-4. Store a Database Connection String Securely .............. 344

9-5. Execute a SQL Command or Stored Procedure .............. 347

9-6. Use Parameters in a SQL Command or Stored Procedure ........ 351

9-7. Process the Results of a SQL Query Using a Data Reader . . . . . . 355

9-8. Obtain an XML Document from a SQL Server Query .......... 358

9-9. Perform Asynchronous Database Operations

Against SQL Server ............................ 362

9-10. Write Database-Independent Code $\ldots \ldots \ldots \ldots \ldots \ldots \ldots . \ldots . \ldots . \ldots 6$

9-11. Discover All Instances of SQL Server on Your Network ........ 370

CHAPTER 10 Networking and Remoting $\ldots \ldots \ldots \ldots \ldots \ldots \ldots \ldots \ldots$

10-1. Obtain Information About the Local Network Interface ....... 374

10-2. Detect Changes in Network Connectivity $\ldots \ldots \ldots \ldots \ldots . \ldots . \ldots 377$

10-3. Download Data over HTTP or FTP .................... 379 
10-4. Download a File and Process It Using a Stream ............ 382

10-5. Respond to HTTP Requests from Your Application ........... 384

10-6. Get an HTML Page from a Site That

Requires Authentication .......................... 388

10-7. Send E-mail Using SMTP $\ldots \ldots \ldots \ldots \ldots \ldots \ldots \ldots \ldots \ldots \ldots \ldots \ldots$

10-8. Resolve a Host Name to an IP Address .................. 394

$10-9$. Ping an IP Address . . . . . . . . . . . . . . . . . . . 396

10-10. Communicate Using TCP ...................... 398

10-11. Create a Multithreaded TCP Server That Supports

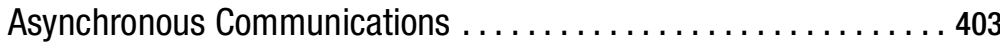

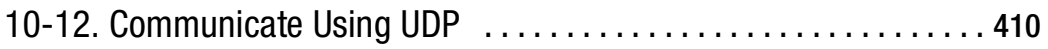

10-13. Avoid Hard-Coding the Web Service URL .............. 413

10-14. Set Authentication Credentials for a Web Service ......... 415

10-15. Call a Web Method Asynchronously ................. 418

10-16. Make an 0bject Remotable ...................... 420

10-17. Register All the Remotable Classes in an Assembly . ....... 425

10-18. Host a Remote Object in IIS $\ldots \ldots \ldots \ldots \ldots \ldots \ldots \ldots \ldots . \ldots 27$

10-19. Control the Lifetime of a Remote 0bject .............. 429

10-20. Control Versioning for Remote Objects $\ldots \ldots \ldots \ldots \ldots \ldots \ldots 431$

CHAPTER 11 Security and Cryptography 433

11-1. Allow Partially Trusted Code to Use Your

Strong-Named Assembly $\ldots \ldots \ldots \ldots \ldots \ldots \ldots \ldots \ldots \ldots \ldots \ldots \ldots$

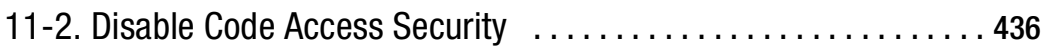

11-3. Disable Execution Permission Checks ................. 438

11-4. Ensure the Runtime Grants Specific Permissions to

Your Assembly . ............................... 440

11-5. Limit the Permissions Granted to Your Assembly .......... 442

11-6. View the Permissions Required

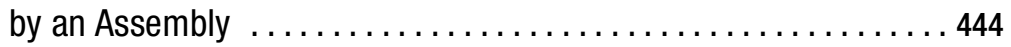

11-7. Determine at Runtime If Your Code Has a

Specific Permission ............................. 446

11-8. Restrict Who Can Extend Your Classes and Override

Class Members ................................ 447

11-9. Inspect an Assembly's Evidence $\ldots \ldots \ldots \ldots \ldots \ldots \ldots . \ldots 449$

11-10. Determine If the Current User Is a Member of a Specific

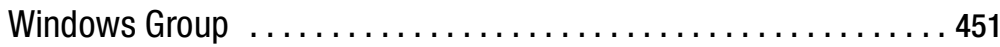

11-11. Restrict Which Users Can Execute Your Code $\ldots \ldots \ldots \ldots . \ldots 455$

11-12. Impersonate a Windows User . . . . . . . . . . . . . . . 458 
11-13. Create a Cryptographically Random Number ........... 462

11-14. Calculate the Hash Code of a Password ............... 463

11-15. Calculate the Hash Code of a File .................. 466

$11-16$. Verify a Hash Code ................................. 469

11-17. Ensure Data Integrity Using a Keyed Hash Code $\ldots \ldots \ldots \ldots . . .471$

11-18. Work with Security-Sensitive Strings in Memory ........... 474

11-19. Encrypt and Decrypt Data Using the Data Protection API . . . . 477

CHAPTER 12 Unmanaged Code Interoperability $\ldots \ldots \ldots \ldots \ldots \ldots \ldots 1$

12-1. Call a Function in an Unmanaged DLL . ................ 481

12-2. Get the Handle for a Control, Window, or File ............ 484

12-3. Call an Unmanaged Function That Uses a Structure . ........ 486

12-4. Call an Unmanaged Function That Uses a Callback .......... 489

12-5. Retrieve Unmanaged Error Information . ............... 491

12-6. Use a COM Component in a .NET Client . ............... 493

12-7. Release a COM Component Quickly .................. 495

12-8. Use 0ptional Parameters . ........................ 496

12-9. Use an ActiveX Control in a .NET Client ................. 497

12-10. Expose a .NET Component to COM .................. 499

CHAPTER 13 Commonly Used Interfaces and Patterns $\ldots \ldots \ldots \ldots . \ldots 501$

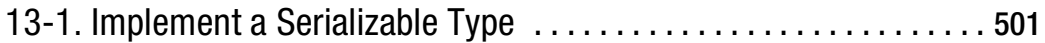

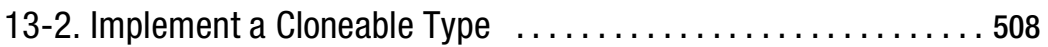

13-3. Implement a Comparable Type . ................... 512

13-4. Implement an Enumerable Type Using a Custom Iterator . . . . . 517

13-5. Implement a Disposable Class . ..................... 523

13-6. Implement a Formattable Type . ................... 527

13-7. Implement a Custom Exception Class ................. 531

13-8. Implement a Custom Event Argument . . . . . . . . . . . . . 535

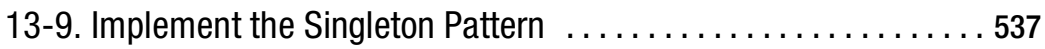

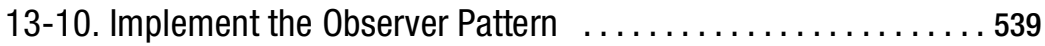

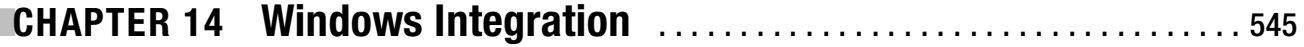

14-1. Access Runtime Environment Information $\ldots \ldots \ldots \ldots \ldots . \ldots 545$

14-2. Retrieve the Value of an Environment Variable ............. 549

14-3. Write an Event to the Windows Event Log ............... 550

14-4. Read and Write to the Windows Registry $\ldots \ldots \ldots \ldots \ldots \ldots 53$ 
14-5. Search the Windows Registry $\ldots \ldots \ldots \ldots \ldots \ldots \ldots \ldots \ldots \ldots 56$

14-6. Create a Windows Service $\ldots \ldots \ldots \ldots \ldots \ldots \ldots \ldots \ldots \ldots . \ldots \ldots$

14-7. Create a Windows Service Installer .................. 564

14-8. Create a Shortcut on the Desktop or Start Menu .......... 567

CHAPTER 15 Language Integrated Query (LINQ) $\ldots \ldots \ldots \ldots \ldots \ldots \ldots 71$

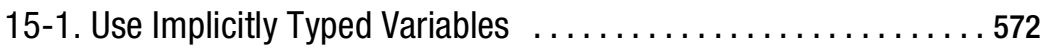

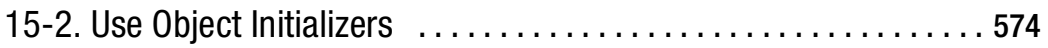

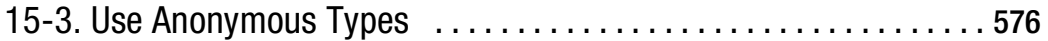

15-4. Create Extension Methods $\ldots \ldots \ldots \ldots \ldots \ldots \ldots \ldots \ldots \ldots \ldots$

15-5. Query an IEnumerable(Of T) Collection ................ 579

$15-6$. Query a Nongeneric Collection ...................... 584

15-7. Sort Data Using LINQ ......................... 585

15-8. Filter Data Using LINQ $\ldots \ldots \ldots \ldots \ldots \ldots \ldots \ldots \ldots \ldots \ldots . \ldots \ldots 9$

15-9. Query Data from Multiple Collections $\ldots \ldots \ldots \ldots \ldots \ldots \ldots 59$

15-10. Perform Aggregate 0perations on Collections ............ 594

15-11. Retrieve a Subset of Data from a Collection ............. 598

15-12. Display Collection Data Using Paging $\ldots \ldots \ldots \ldots \ldots \ldots \ldots 60$

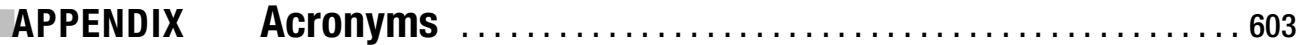

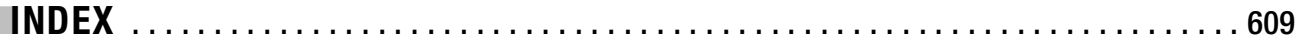




\section{About the Authors}
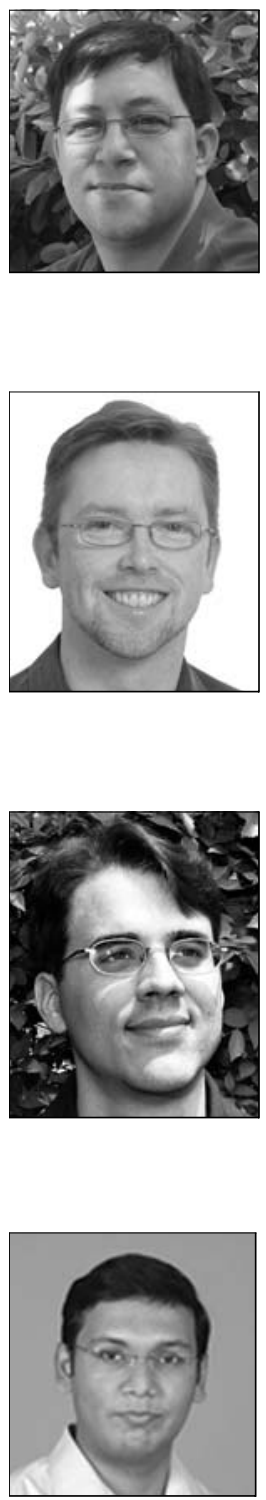

TODD HERMAN works for Northrop Grumman developing software for the Department of State. He has been programming since he received his first computer, a Commodore 64, on his eleventh birthday. His experience ranges from developing data-entry software in FoxPro for a water research laboratory to writing biometric applications in Visual Basic for NEC. He currently lives in Virginia with his wife and children, and spends his free time programming, playing games, and watching the Sci-Fi channel.

ALLEN JONES has 15 years of experience covering a wide range of IT disciplines in a variety of sectors; however, his true passion has always been software development. Allen is currently Director of Product Development at SmithBayes, a UK-based firm that develops high-end, strategic-decision-support software derived from technology used in Formula 1 motor racing.

MATTHEW MACDONALD is an author, educator, and Microsoft MVP. He is a regular contributor to programming journals and the author of more than a dozen books about .NET programming, including Pro.NET 2.0 Windows Forms and Custom Controls in C\#, Pro ASP.NET 2.0, and Pro WPF (each published by Apress). In a dimly remembered past life, he studied English literature and theoretical physics.

RAKESH RAJAN coauthored an earlier book on which this book is based (Visual C\# 2005 Recipes, Apress, 2006). Sadly, Rakesh passed away in 2006 . He was a Microsoft MVP in C\# and an MCSD in .NET. As a software engineer from India, Rakesh worked with US Technology at Technopark, Trivandrum in Kerala. 


\section{About the Technical Reviewer}

DAMIEN FOGGON is a freelance programmer and technical author based in Newcastle, England. He is technical director of Thing-E Ltd., a company specializing in the development of dynamic web solutions for the education sector, and founder of Littlepond Ltd. He started out working for BT in the UK before moving on to progressively smaller companies, finally founding his own company. Now he can work on all the cool new technologies, rather than the massive monolithic developments that still exist out there.

Damien is the author of Beginning ASP.NET 2.0 Databases: From Novice to Professional (Apress, 2006). He can be reached at http://www.littlepond.co.uk. 


\section{Acknowledgments}

first want to thank Richard Guidorizzi for reaffirming my thoughts regarding writing a book. Furthermore, I must thank Ewan Buckingham for seeing something in my e-mail and offering me the opportunity to write this. Finally, I must also extend my thanks and appreciation to Damien Foggon, my technical reviewer. His comments and suggestions forced me to dig deeper into myself and the material to make this a book to be proud of.

Todd Herman 


\section{Introduction}

A

ttempting to learn all there is to know about developing VB .NET applications using the Microsoft .NET Framework would be an incredibly daunting task. For most of us, the easiest and best approach is to dive in and start writing code. We learn through testing and experimentation, and when we run into the unknown, we search the Internet or grab a book to assist with the current subject.

Visual Basic 2005 Recipes is not a book that attempts to teach you about the inner workings of a specific subject. It is a resource book that should sit near you as you program, where you can quickly use it to reference what you need.

As you are settled in front of your computer working, you will inevitably run into a situation where you need a little guidance, as all of us do from time to time. The subject matter in this book is so comprehensive that you are bound to find at least one recipe that will fit the bill whenever you need that nudge in the right direction.

This book will not teach you everything you need know about developing VB .NET applications in Visual Studio 2005, but it will be invaluable as a stepping stone. Use the recipes as you need them, to help move your development projects along or to give you a starting point for your own experimentation.

Note This book is based on a previously published book called Visual C\# 2005 Recipes. All the contents were converted to Visual Basic and updated to reflect some VB-specific features and functionality. While the recipes all target .NET Framework 2.0, many of the recipes will still work on .NET Framework 1.1. Where a recipe uses functionality specific to .NET Framework 2.0, comments were added to present possible alternatives for using .NET Framework 1.1. Furthermore, as we are on the cusp of a new version of the .NET Framework (3.5) and Visual Studio (code-named Orcas), Chapter 15 covers a new and remarkable feature known as Language Integrated Query (LINQ). 